Article

\title{
An Injectable Hyaluronan-Methylcellulose (HAMC) Hydrogel Combined with Wharton's Jelly-Derived Mesenchymal Stromal Cells (WJ-MSCs) Promotes Degenerative Disc Repair
}

\author{
Un Yong Choi ${ }^{1,+}{ }^{1}$, Hari Prasad Joshi ${ }^{1,+}$, Samantha Payne ${ }^{2,+}$, Kyoung Tae Kim ${ }^{3,4}{ }^{\oplus}$, \\ Jae Won Kyung ${ }^{1}$, Hyemin Choi ${ }^{1}$, Michael J. Cooke ${ }^{5,6}$, Su Yeon Kwon ${ }^{1}$, Eun Ji Roh ${ }^{1}$, Seil Sohn ${ }^{1}$, \\ Molly S. Shoichet ${ }^{5,6}$ and Inbo Han 1,*D \\ 1 Department of Neurosurgery, CHA Bundang Medical Center, CHA University School of medicine, \\ Seongnam-si 13496, Korea; nschoiuy@gmail.com (U.Y.C.); hariprasadjoshi10@gmail.com (H.P.J.); \\ kyungjaewon88@gmail.com (J.W.K.); littlechoi88@gmail.com (H.C.); syunkwon@naver.com (S.Y.K.); \\ morolro@naver.com (E.J.R.); sisohn@cha.ac.kr (S.S.) \\ 2 Department of Biomedical Engineering, Tufts University, Medford, MA 02155, USA; \\ Samantha.payne@tufts.edu \\ 3 Department of Neurosurgery, School of Medicine, Kyungpook National University, Daegu 41944, Korea; \\ nskimkt7@gmail.com \\ 4 Department of Neurosurgery, Kyungpook National University Hospital, Daegu 41404, Korea \\ 5 Department of Chemical Engineering and Applied Chemistry, University of Toronto, 200 College Street, \\ Toronto, ON M5S 3E5, Canada; mikejcooke@amacathera.ca (M.J.C.); molly.shoichet@utoronto.ca (M.S.S.) \\ 6 Donnelly Centre, University of Toronto, 160 College Street, Toronto, ON M5S 3E1, Canada \\ * Correspondence: hanib@cha.ac.kr \\ $\dagger$ These authors contributed equally to this work.
}

Received: 5 September 2020; Accepted: 2 October 2020; Published: 7 October 2020

check for updates

\begin{abstract}
Intervertebral disc (IVD) degeneration is one of the predominant causes of chronic low back pain (LBP), which is a leading cause of disability worldwide. Despite substantial progress in cell therapy for the treatment of IVD degeneration, significant challenges remain for clinical application. Here, we investigated the effectiveness of hyaluronan-methylcellulose (HAMC) hydrogels loaded with Wharton's Jelly-derived mesenchymal stromal cell (WJ-MSCs) in vitro and in a rat coccygeal IVD degeneration model. Following induction of injury-induced IVD degeneration, female Sprague-Dawley rats were randomized into four groups to undergo a single intradiscal injection of the following: (1) phosphate buffered saline (PBS) vehicle, (2) HAMC, (3) WJ-MSCs $\left(2 \times 10^{4}\right.$ cells), and (4) WJ-MSCs-loaded HAMC (WJ-MSCs/HAMC) ( $n=10 /$ each group). Coccygeal discs were removed following sacrifice 6 weeks after implantation for radiologic and histologic analysis. We confirmed previous findings that encapsulation in HAMC increases the viability of WJ-MSCs for disc repair. The HAMC gel maintained significant cell viability in vitro. In addition, combined implantation of WJ-MSCs and HAMC significantly promoted degenerative disc repair compared to WJ-MSCs alone, presumably by improving nucleus pulposus cells viability and decreasing extracellular matrix degradation. Our results suggest that WJ-MSCs-loaded HAMC promotes IVD repair more effectively than cell injection alone and supports the potential clinical use of HAMC for cell delivery to arrest IVD degeneration or to promote IVD regeneration.
\end{abstract}

Keywords: mesenchymal stromal cell; Wharton jelly; hyaluronic acid; methylcellulose; intervertebral disc degeneration; regeneration; extracellular matrix 


\section{Introduction}

Chronic low back pain (LBP) is the leading cause of disability worldwide and the social and economic impact is enormous [1]. Not all degenerated discs exhibit chronic LBP, however, intravertebral disc (IVD) degeneration is considered as a major cause of chronic LBP [1-4]. IVD degeneration is a complex and multifactorial process, influenced by genetic, nutritional, and mechanical factors [5]. As the stage of degeneration progresses, the production of pro-inflammatory molecules, including tumor necrosis factor (TNF)- $\alpha$, interferon gamma (IFN- $\gamma$ ), and interleukin (IL-1 $\beta$, IL-6, IL- $1 \alpha$ and IL-2) increases [5]. These molecules can be produced by both IVD cells and immune cells, such as macrophages [6], and are known to be associated with discogenic back pain [6]. IVD degeneration is characterized by the loss of IVD cells and extracellular matrix (ECM) such as aggrecan and collagen type II, with the upregulation of matrix metalloproteinases (MMPs) and inflammatory mediators leading to progressive and irreversible damage of IVD structure [1,3,4,6-8]. Eventually, IVD degeneration can lead to the onset of additional spinal conditions, including disc herniation, spinal stenosis, facet joint osteoarthritis, and spondylolisthesis $[1,3,9,10]$. Current conservative and surgical treatments focus on symptomatic relief rather than arresting degeneration or restoring the structure and function of the degenerated disc $[3,11]$. Furthermore, the effect of surgical treatments may be temporary, and recurrent or persistent LBP can develop [5]. Therefore, there is a strong clinical demand for the development of regenerative therapy to repair degenerated disc or arrest IVD degeneration at earlier stages $[1,4]$. Among the biological approaches for disc repair, cell therapy has gained interest because it offers disc regeneration potential [12-14]. Especially, mesenchymal stromal cells (MSCs) have been considered as a potentially ideal cell source for IVD regeneration as MSCs display broad immunomodulatory properties and the ability to differentiate into cartilage [5]. Several clinical studies on IVD cell therapies reported that MSC implantation can regenerate the degenerated disc and cure discogenic back pain $[5,12]$.

Despite some positive outcome of MSC-based therapies, the strength of the evidence for use of MSCs in IVD degeneration is low, due to the significant risk of bias, small sample size, and lack of a control group in clinical trials [5]. Furthermore, for the successful development of MSC-based cell therapy for disc repair, there are still many obstacles to overcome such as the inflammatory milieu of the degenerated disc. For successful regeneration of the IVD, the selection of a proper cell type and source is crucial. Wharton's jelly-derived MSCs (WJ-MSCs) have gained significant interest and attention for clinical application because of their hypoimmunogenicity and immunomodulatory potential compared with MSCs from other sources [4,15-18]. WJ-MSCs exhibit very low expression of human leukocyte antigen (HLA) class I and do not express class II (HLA-DR) as well as co-stimulatory antigens such as CD86, CD80, the key players to activate B cell and T cell $[19,20]$. Furthermore, WJ-MSCs synthesize extensive amounts of tolerogenic IL-10, up-regulate TGF- $\beta$ and express HLA-G [21]. Their low cellular immunogenicity is advantageous for allogeneic and xenogeneic transplantation. WJ-MSCs also possess immunomodulatory potential $[18,22]$. Their immunomodulatory mechanism can be explained as: upregulation of negative co-stimulatory ligands; secretion of immunosuppressive soluble factors; generation of memory cells; cell fusion to escape recognition; immune avoidance mechanisms specific to fetal-maternal interface; attenuation of antigen-presenting cell functions; altered migration of immune cells; T cell energy apoptosis tolerance $[18,22]$. Thus, WJ-MSCs have been considered to be optimal candidates for cellular therapies in allogenic transplantation and used for treatment of various diseases (e.g., cancer, chronic liver disease, cardiovascular diseases, cartilage and tendon injury, immune mediated disorders, such graft versus host disease and systemic lupus erythematosus) [18,22].

Another strategy for successful IVD regeneration, biomaterial technology has gained increasing attention as a vehicle for cell delivery to promote the viability and differentiation of MSCs by providing a three-dimensional (3D) microenvironment and to prevent cell leakage and reduce the risk of osteophyte formation $[4,12,14]$. In particular, an injectable, biocompatible, and biodegradable hydrogel scaffold composed of hyaluronan (HA) and methylcellulose (MC) (HAMC) is of particular interest for therapeutic cell delivery. The HAMC hydrogel is biodegradable, water soluble and provides a three-dimensional (3D) microenvironment for the cells, thereby enhancing cell viability (Figure 1A) [23,24]. Over the last 
decade, Shoichet lab has developed HAMC for various neurological disease models, such as ischemic stroke [24], retinal degeneration [25] and spinal cord injury $[23,26]$. HAMC spatially localizes the drug or cells of interest at the site of delivery and promotes short-term controlled release of drugs to the central nervous system (CNS), with a degradation time of approximately 3 to 7 days in vivo [27-30]. Importantly, it shows low immunoreactivity upon delivery and due to the property of inverse thermal gelling, cells can be mixed into HAMC at room temperature and when delivered it will increase in viscosity to retain cells at the site of injection [31].

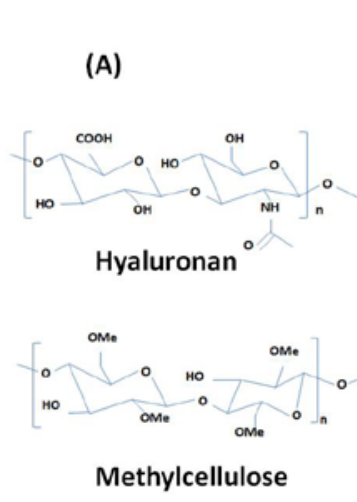

(B)
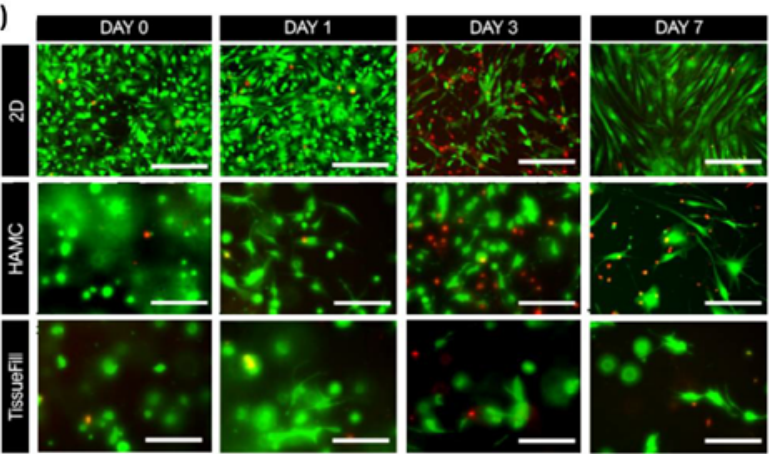

(c)

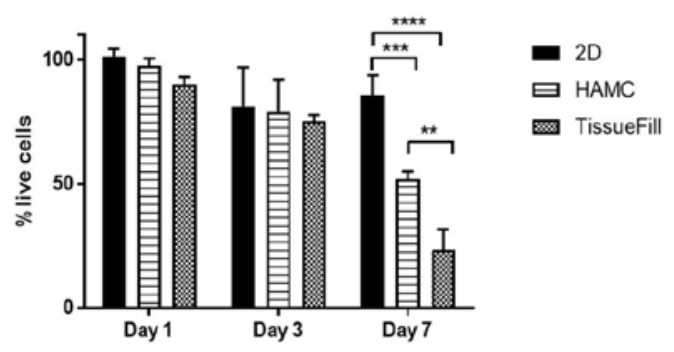

Figure 1. HAMC supports 3D WJ-MSCs survival in vitro. Cells were cultured in 3D HAMC and compared to 2D plastic culture or 3D TissueFill. Cell viability was significantly higher for cells cultured in HAMC compared to TissueFill. (A) Molecular structure of hyaluronan (HA) and methyl cellulose (MC). (B) Representative immunofluorescence images for live (green) and dead (red) assay in 2D, HAMC and TissueFill at day 0, day 1, day 3 and day 7 following WJ-MSCs culture. (C) Quantitative bar graph illustrating the percentage of live cells in 2D, HAMC and TissueFill at day 0, day 1, day 3 and day 7 following WJ-MSC culture. After 7 days of culture HAMC had significantly higher viability than TissueFill. Mean $+\mathrm{SD}, n=3$ biological replicates. Two-way ANOVA with Tukey's post hoc, ${ }^{* *} p<0.01$, ${ }^{* * *} p<0.001,{ }^{* * * *} p<0.0001$. Scale bar $=50 \mu \mathrm{m}$.

In our previous study, we first confirmed that biomaterial loaded with human WJ-MSCs has good regenerative potential for IVD repair in a rabbit model of disc degeneration [4]. WJ-MSCs were chosen because of several advantages for clinical use, such as robust immunomodulatory properties $[4,15,16]$. Additionally, we chose TissueFill (cross-linked HA derivatives; CHA Meditech Co., Ltd., Dajeon, Korea), a commercially available dermal filler as a cell carrier because there was no commercialized biomaterial for IVD repair. In the present study, we have investigated whether WJ-MSCs-loaded HAMC could induce IVD repair more effectively than cell alone injections and support the potential clinical use of HAMC for cell delivery for IVD repair.

\section{Results}

\subsection{Quality Control of WJ-MSCS}

The WJ-MSCs in the second, fourth, and seventh generations showed a spindle fibroblast-like shape (Figure S1A). After induction with adipogenic medium, WJ-MSCs gradually changed from fibroblast-like cells to flattened cells, and lipid droplets accumulated within them. The adipogenic 
differentiated MSCs were visualized by staining with Oil red-O on day 15. After incubation with osteogenic or chondrogenic medium for 15 days, MSCs were positive for alizarin red and alcian blue staining, respectively (Figure S1B). The cell population doubling time was calculated three times. The cell doubling time in the second, fourth, and seventh generation were $22.35 \pm 0.53 \mathrm{~h}$, $17.93 \pm 0.72 \mathrm{~h}$, and $18.63 \pm 1.1 \mathrm{~h}$, respectively, and the difference between the two groups was not significant (Figure S1C). Flow cytometry analysis of expressed surface antigens showed that these cells were uniformly positive for CD44, CD73, CD105, and CD90, and negative for the hematopoietic lineage markers CD45 (Figure S1D). No structural or numerical chromosomal abnormalities were found in karyotype analyses of the cells until P14.

\subsection{HAMC Promotes 3D In Vitro Survival of WJ-MSCS}

To determine if HAMC is a suitable delivery vehicle for the injection of WJ-MSCs into the IVD space, we quantified cell viability following $3 \mathrm{D}$ culture in $0.5 \%$ weight per volume $(w / v)$ HAMC and compared it to TissueFill, a commercially available crosslinked hyaluronan hydrogel (Figure 1B). After 7 days, we observed that cell viability was significantly increased with culture in HAMC compared to TissueFill, $52 \%$ and $24 \%$ viability, respectively (Figure $1 \mathrm{~B}, \mathrm{C}$ ).

\subsection{HAMC Supports Improved Post-Injection WJ-MSCs Viability Compared to TissueFill}

The injection of cells for transplantation through a 26-gauge $(G)$ needle can cause cell death due to the shear forces cells will experience as they pass from the syringe to the needle [32-35], which can be partially negated with the use of a biomaterial for the cell carrier [36]. To determine if WJ-MSC viability is supported by HAMC during the injection process, we recapitulated the cell delivery protocol in vitro and measured acute cell viability following injection (Figure 2A,B). Cells were encapsulated in $0.5 \%$ $w / v$ HAMC, $1 \%$ TissueFill, or cell medium and injected through a $26 \mathrm{G}$ needle as per the in vivo delivery protocol into a 96-well plastic culture plate (Figure 2A,B). Cell viability was assessed immediately following injection. It was observed that viability decreases significantly following injection in all groups (HAMC, TissueFill, or medium) compared to no injection group, but that cells encapsulated in TissueFill had significantly reduced viability compared to HAMC or cell medium alone (Figure 2C (i) and (ii)). This suggests that HAMC is a more suitable cell delivery vehicle to maintain viability in comparison to TissueFill. 
(A)

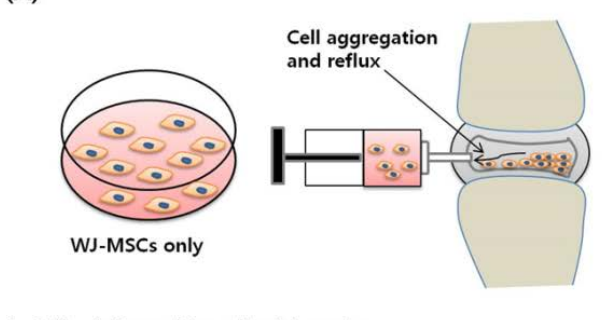

1. Cells delivered in a liquid carrier

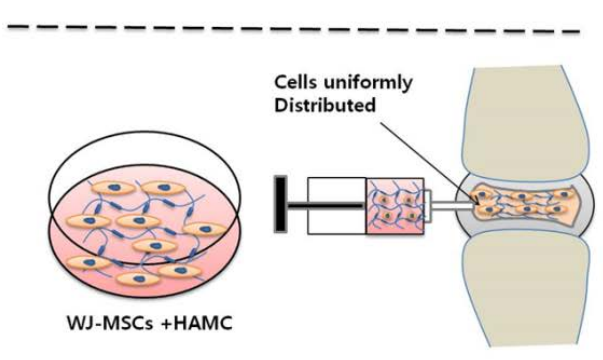

2. Cells delivered within a HAMC carrier

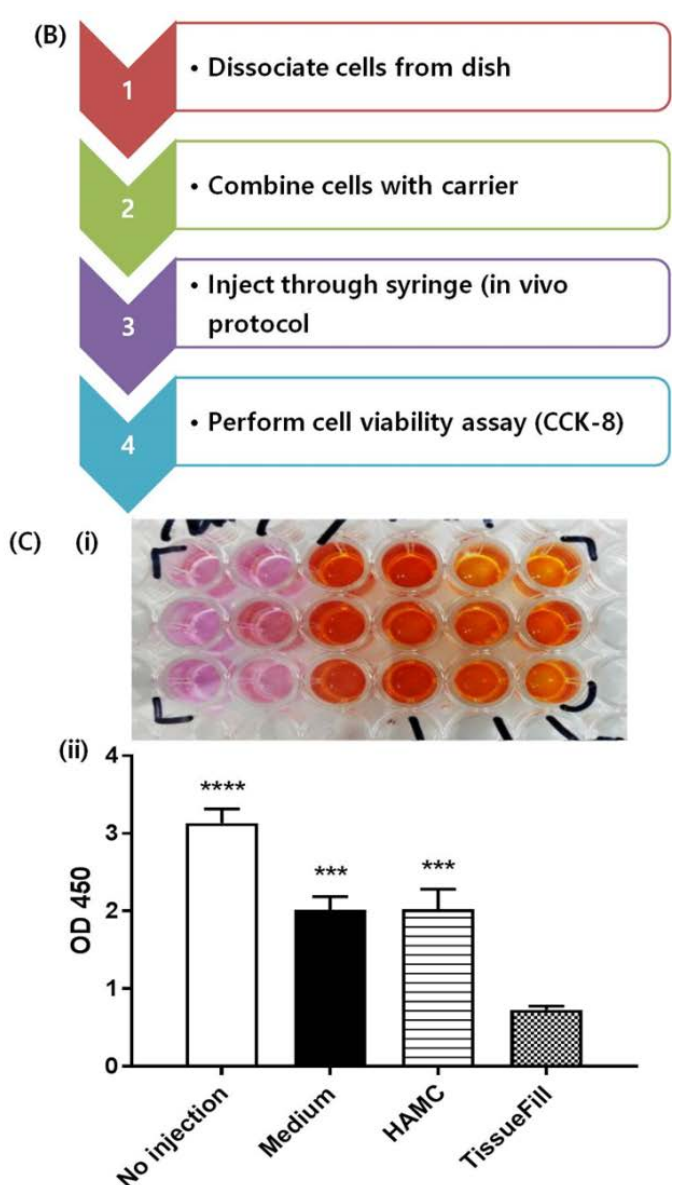

Figure 2. Encapsulation in HAMC maintains post-injection viability compared to TissueFill. WJ-MSCs were encapsulated in different vehicles and injected through a fine-gauge needle to assess post-injection viability. (A) Schematic illustrating the comparison of WJ-MSC stability and viability between HAMC-encapsulated, and non-encapsulated WJ-MSCs post-injection. (B) Stepwise post-injection procedure. (C) (i) Representative CCK-8 assay, (ii) quantitative result after CCK-8 assay, compareing the cell viability of WJ-MSCs in medium alone, TissueFill or HAMC. Cells injected in medium or HAMC retained significantly higher viability compared to TissueFill. Mean $+\mathrm{SD}, n=3$ biological replicates. One-way ANOVA with Tukey's post hoc, ${ }^{* * *} p<0.001,{ }^{* * * *} p<0.0001$ compared to TissueFill group.

\subsection{HAMC and WJ-MSCs Transplantation Restores the Disc Anatomy and Water Content of IVDs Following IVD Degeneration}

The in-vivo IVD degeneration study was conducted using a well-established rat tail coccygeal needle puncture (Co 6-7 and Co 7-8) model. This model is commonly used due to easy accessibility of this area in rats and reported reproducibility [37]. After 2 weeks after needle injury, we injected PBS, WJ-MSCs, or WJ-MSCs-loaded HAMC $\left(2 \times 10^{4}\right.$ WJ-MSCs in $1 \mu \mathrm{L}+1 \mu \mathrm{L}$ of $1 \% w / v$ HAMC $)$ into the degenerated disc (Co 6-7 and Co 7-8) and performed MRI at six weeks after implantation (Figure 3A). An MRI index was calculated for control disc (no injury), vehicle (PBS treated), HAMC, WJ-MSCs, and HAMC + WJ-MSCs-treated discs (Figure 3B). On coronal T2-weighted MRI, distinct and bright signal intensity of the disc was observed in the control healthy disc (red arrows, Co 5-6) (MRI index: $142.15 \%$ ). Vehicle-injected disc showed significant decrease in signal intensity and MRI index. However, HAMC/WJ-MSCs-treated discs revealed a significant increase in both signal intensity and MRI index (104.62\%) (Figure 3A,B).Whereas, the MRI indices for discs treated with HAMC scaffold or WJ_MSCs only were $39.38 \%$ and $61.28 \%$, respectively; almost 3-fold and 2-fold lower, respectively, than the combined injection of HAMC and WJ-MSCs. By contrast, the MRI index for vehicle-treated injured discs was found to be $14.31 \%$, 4-fold lower than that of WJ-MSCs-loaded 
HAMC-treated discs. Overall, the result indicates that a significantly higher hydration level was retained in HAMC/WJ-MSCs-treated discs compared to HAMC only and WJ-MSCs only implanted discs. Moreover, MRI findings demonstrated that gross anatomical structure and MRI intensity of HAMC + WJ-MSCs injected disc was similar to normal (control) (Figure 3A), indicating enhanced disc regeneration by HAMC + WJ-MSCs.
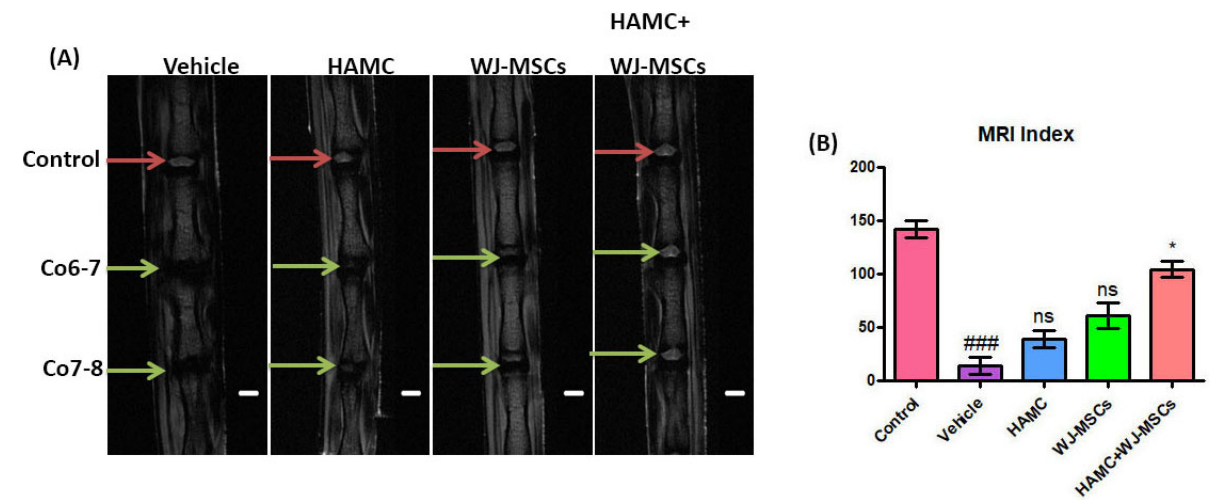

(C)
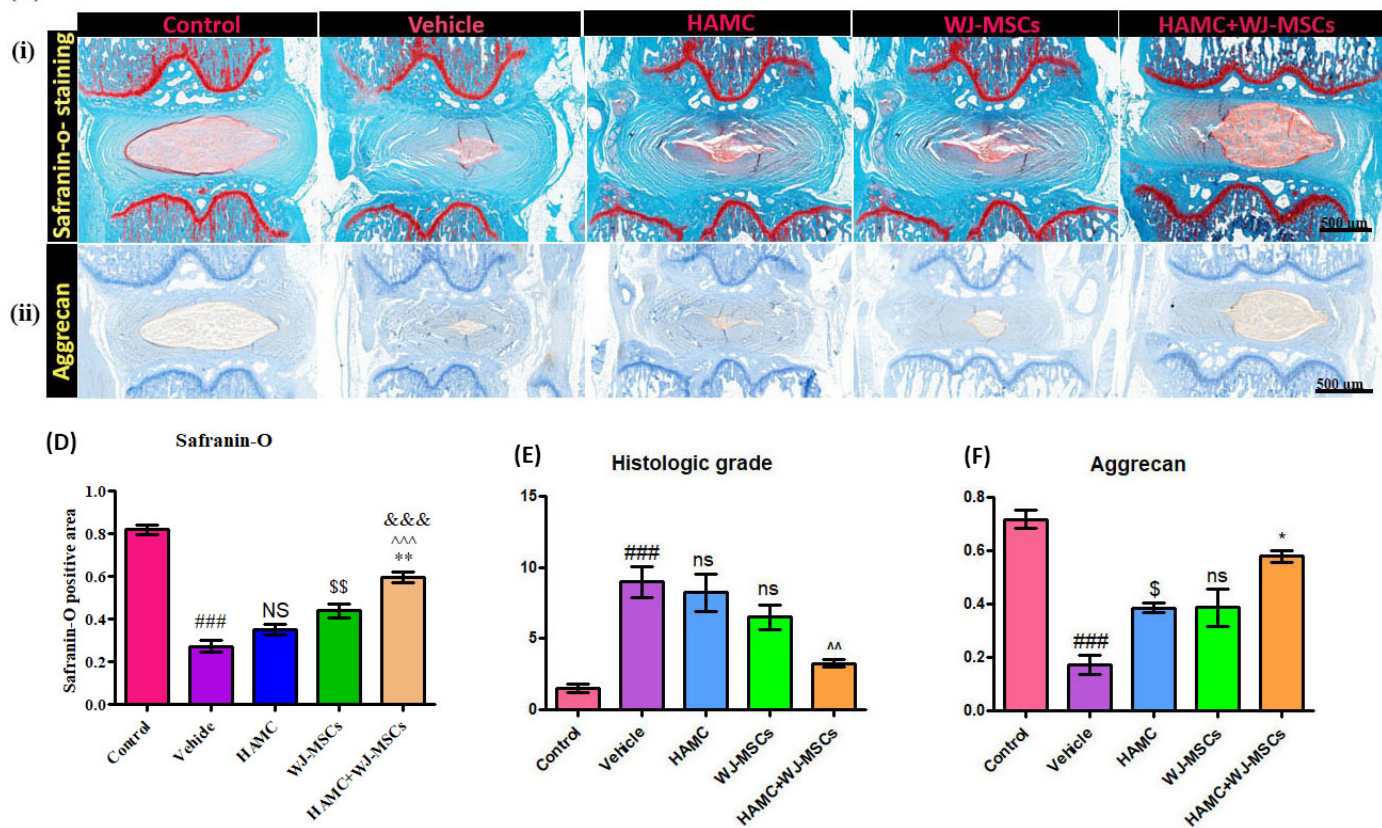

Figure 3. WJ-MSCs-loaded HAMC injection promotes repair of the degenerated disc (A) Representative T2-weighted MRI of the coccygeal discs: control, vehicle-treated, HAMC-treated, WJ-MSCs only treated, and HAM + CWJ-MSC-treated disc (coronal view) (red arrow = control, green arrow = needle-punctured disc). (B) MRI index for discs of each group. Mean $\pm \mathrm{SEM},(n=3)$, one-way ANOVA followed by Tukey's post-test. \#\# $p<0.05$ (vehicle vs. control), ${ }^{*} p<0.05$ (HAMC + WJ-MSCs vs. WJ-MSCs); ns = non-significant. MRI performed at 6 weeks after implantation showed the best restoration of disc anatomy and water content of the disc in HAMC/WJ-MSC-implanted discs. (C) (i) and (D) Representative images of safranin-O staining and quantitative safranin-O positive area, revealed the highest preservation of disc structure in HAMC/WJ-MSCs-injected disc $(\times 200)$. Mean \pm SEM, $(n=3)$, one-way ANOVA followed by Tukey's post-test. ${ }^{\# \# ~} p<0.001$ (vehicle vs. Sham), $\$ \$ p<0.01$ (WJ-MSCs vs. HAMC), ${ }^{* *} p<0.01$ (HAMC + WJ-MSCs vs. WJ-MSCs), ${ }^{\wedge} p<0.001$ (HAMC + WJ-MSCs vs. HAMC), and \&\&\& $p<0.001$ (HAMC + WJ-MSCs vs. vehicle). (E) The quantitative histological score and (C) (ii) and (F) immunoreactivity for aggrecan showed the highest expression in HAMC/WJ-MSCs-implanted discs. Mean \pm SEM, $(n=3)$, one-way ANOVA followed by Tukey's post-test. ${ }^{\# \# \# ~} p<0.001$ (vehicle vs. Sham), ${ }^{\$} p<0.05$ (HAMC vs. vehicle), ${ }^{*} p<0.05$ (HAMC + WJ-MSCs vs. WJ-MSCs). 


\subsection{HAMC and WJ-MSCs Maintains the Proteoglycan Content and Disc Structure}

After MRI analysis, all isolated discs were evaluated by histological analysis. Any abnormalities such as osteophytes formation were absent in all experimental animals. Histological analysis by safranin-O staining and histologic scores demonstrated the preservation of a proteoglycan matrix in the nucleus pulposus (NP) of the disc and histologic structures of the disc. Our results show that Safranin-O-staining intensity of the NP was significantly reduced, and histologic grade was significantly increased after disc injury (Figure 3C (i) and D), indicating decreased proteoglycan content and less preservation of disc structure. By contrast, the HAMC/WJ-MSCs-treated discs showed significantly increased intensity of safranin-O staining and lowest histologic score compared to vehicle, HAMC and WJ-MSCs only injected discs, suggesting that it was able to maintain integrity of disc matrix structure (Figure 3C (i), D and E). In addition to that, HAMC/WJ-MSCs-treated discs revealed a significant increase in aggrecan (a major component of the disc matrix) content in the NP tissue following disc degeneration (Figure $3 \mathrm{C}$ (ii) and F). In contrast, dramatically diminished aggrecan expression was observed in vehicle injected discs (Figure 3C (ii) and F) (\#\#\# $p<0.001$ (vehicle vs. Sham), $\$ p<0.05$ (HAMC vs. vehicle), ${ }^{*} p<0.05$ (HAMC/WJ-MSCs vs. WJ-MSCs)). Therefore, the marked histo-morphological difference between HAMC/WJ-MSCs and other groups tested demonstrates the potential of HAMC/WJ-MSCs for viable disc repair.

\subsection{HAMC and WJ-MSCs Restore the Matrix Proteins and Downregulate Catabolic Enzymes}

One of the main consequences of IVD degeneration is the degradation of ECMs such as aggrecan and type II collagen. Herein, extensively less expression of collagen type II (a component of the disc NP matrix) was observed in vehicle-injected discs compared to control discs, whereas, its expression was significantly increased in the HAMC/WJ-MSC-injected discs compared to vehicle, HAMC and WJ-MSC only injected discs (Figure 4A (i), (ii) and B). Immunopositivity for both aggrecan and type II was also higher in WJ-MSCs than vehicle and HAMC, showing efficacy of the cells alone, although it did not reach statistical significance (Figure 4A (i), (ii) and B), ${ }^{\# \#} p<0.001$ (vehicle vs. control), ${ }^{\wedge} p<0.05$ (WJ-MSCs vs. HAMC), ${ }^{* *} p<0.01$ (HAMC + WJ-MSCs vs. WJ-MSCs).

MMP-13 plays a role in the degradation of ECM proteins including aggrecan [11,38]. Representative immunostaining for MMP-13 shows that the percentage of immunopositive cells for MMP-13 was significantly increased in vehicle-treated discs (Figure 5A (i), (ii) and B). In contrast, MMP-13 expression was greatly diminished in HAMC/WJ-MSCs-implanted discs (Figure 5A (i), (ii) and B), ${ }^{\# \#} p<0.001$ (vehicle vs. control), ${ }^{* *} p<0.01$ (HAMC + WJ-MSCs vs. WJ-MSCs). 
(A)

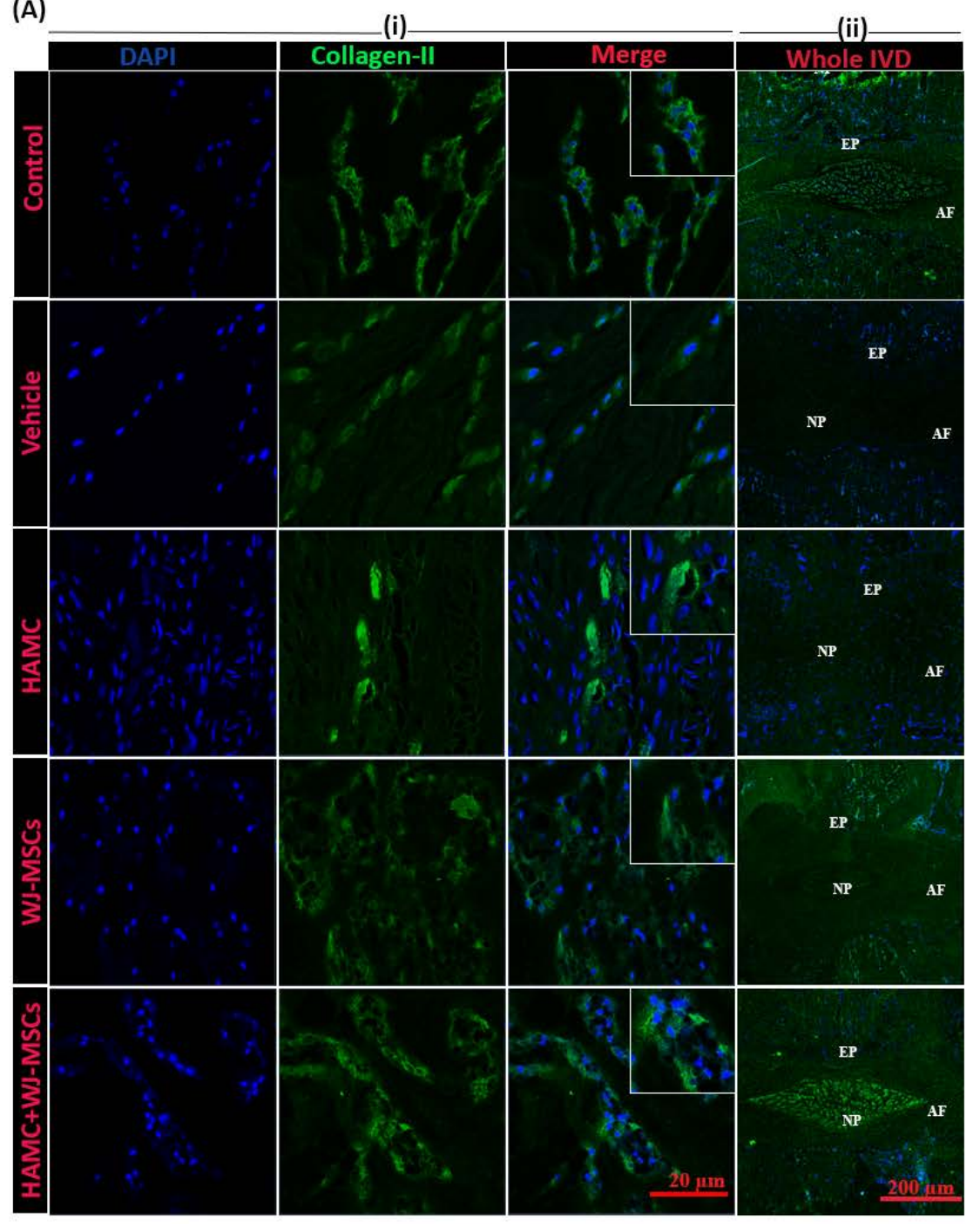

(B)

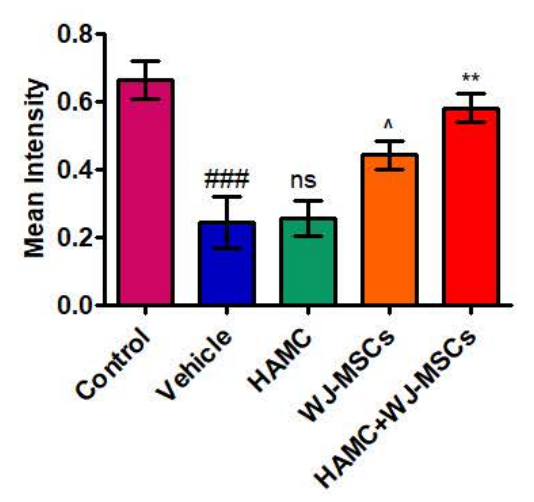

Figure 4. Combined injection of HAMC and WJ-MSC significantly restores collagen type II in degenerated disc. (A) Representative collagen II immunofluorescent staining. (i) Collagen expression at region of interest (ROI) and (ii) full power images demarcating entire disc (nucleus pulposus-NP, annulus fibrosus-AF and end plate-EP), and collagen II expression in whole NP area. (B) Quantitative immunofluorescence intensity for collagen II expression. Mean \pm SEM, $(n=3)$, one-way ANOVA followed by Tukey's post-test. \#\# $p<0.001$ (vehicle vs. control), ^ $p<0.05$ (WJ-MSCs vs. HAMC), ${ }^{* *} p<0.01$ (HAMC + WJ-MSCs vs. WJ-MSCs), ns = non-significant. Combined injection of HAMC and WJ-MSCs significantly preserved the extracellular matrix (ECM) content, collagen type II compared to the vehicle, HAMC, or WJ-MSCs only injected discs. 
(A)

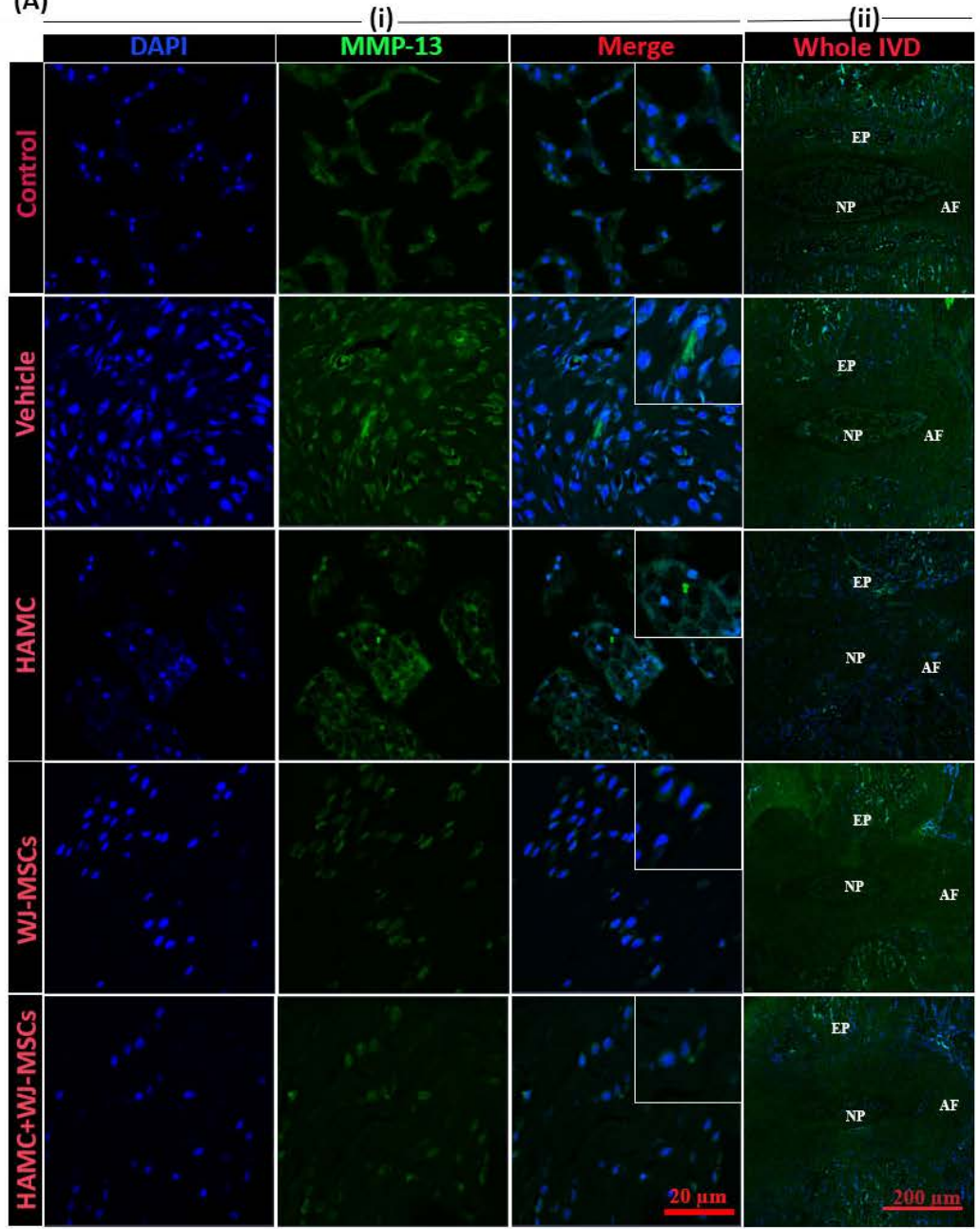

(B)

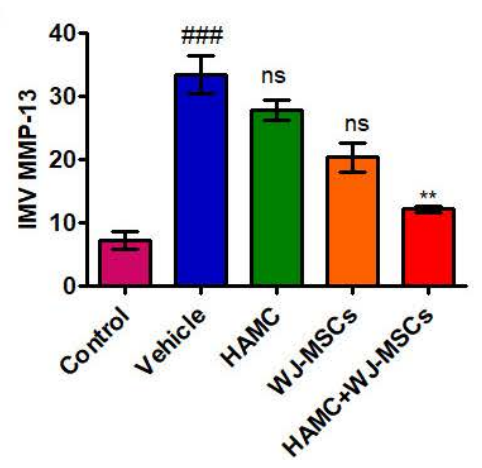

Figure 5. Combined injection of HAMC and WJ-MSC substantially reduced matrix metalloproteinase-13 (MMP-13) expression in degenerated disc. (A) Representative MMP-13 protein levels as determined by immunofluorescence staining. (i) MMP-13 expression (green) at region of interest (ROI), DAPI (blue) and merge signal (ii) full power images (far right panel) demarcating entire disc (nucleus pulposus-NP, annulus fibrosus-AF and end plate-EP), and MMP-13 expression in whole NP area. (B) Quantitative immunofluorescence intensity for MMP-13 expression. Immunopositivity was counted in at least three random low power fields $(\times 40)$ and calculated as mean intensity. Mean \pm SEM, $(n=3)$, one-way ANOVA followed by Tukey's post-test. ${ }^{\# \# \# ~} p<0.001$ (vehicle vs. control), ${ }^{* *} p<0.01$ (HAMC + WJ-MSCs vs. WJ-MSCs). Ns = non-significant. We found that combined injection of HAMC and WJ-MSC significantly diminished the expression of matrix-degrading enzyme, MMP-13. 


\subsection{HAMC and WJ-MSCs Inhibit the mRNA Expression of Pro-Inflammatory Cytokines and} Matrix-Degrading Proteases

Next, we evaluated the various cytokines expression in the degenerated discs. As shown in Figure 6, the RT-qPCR results demonstrate significantly upregulated mRNA expression of inflammatory cytokines, inducible nitric oxide synthase (iNOS) (Figure 6A) (\#\#\# $p<0.001$ vs. control), and matrix-degrading enzymes at 8 weeks following disc injury including: MMP-13 (Figure 6B) (\#\#\# $p<0.001$ vs. control), A disintegrin and metalloproteinase with thrombospondin motifs 4 (Adamts4) (Figure 6C) ( ${ }^{\#} p<0.05$ vs. control), and Cycloxygenase-2 (Cox-2) (Figure 6D) $\left(^{\#} p<0.05\right.$ vs. control) relative to control. By contrast, mRNA expression of $i N O S\left({ }^{* * *} p<0.001\right.$ vs. vehicle), MMP-13 $\left({ }^{* * *} p<0.001\right.$ vs. vehicle), Adamts4 ( ${ }^{* * *} p<0.001$ vs. vehicle) and Cox $-2\left({ }^{* * *} p<0.001\right.$ vs. vehicle) was significantly reduced in discs treated with HAMC/WJ-MSCs as compared to vehicle injected discs, indicating that WJ-MSCs-loaded HAMC prevented ECM degradation by suppressing expression of pro-inflammatory cytokines and matrix-degrading enzymes.

(A)

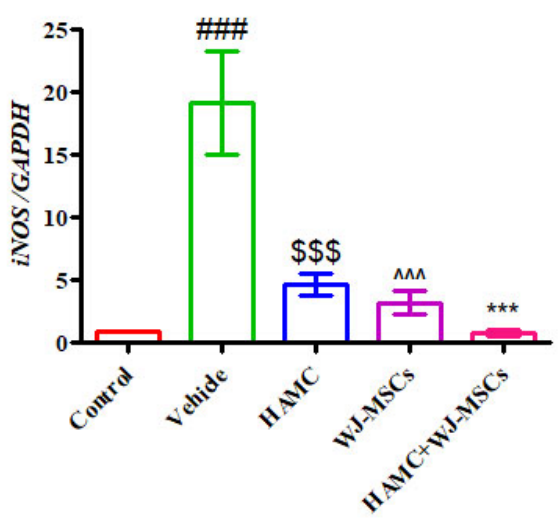

(C)

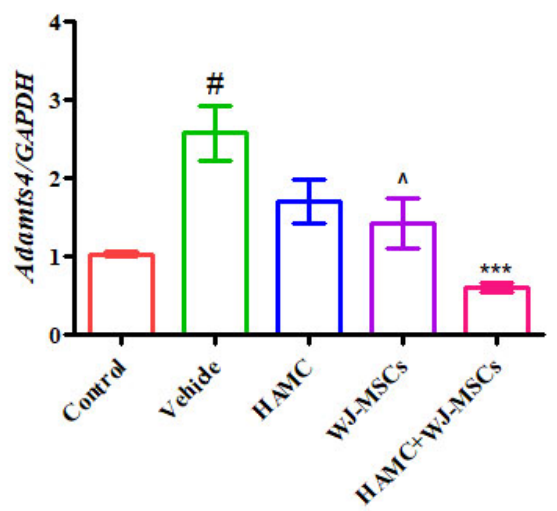

(B)

MMP-13

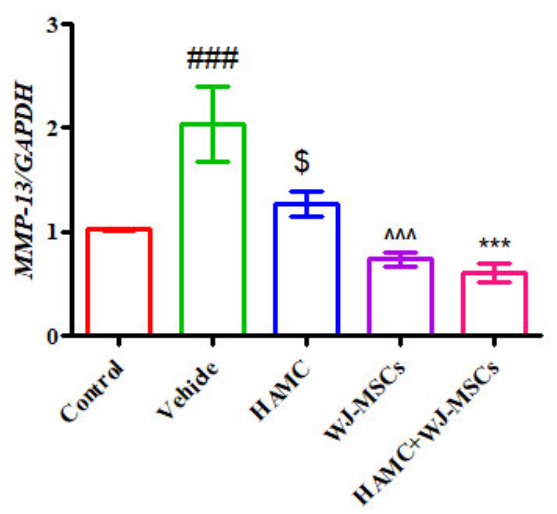

(D)

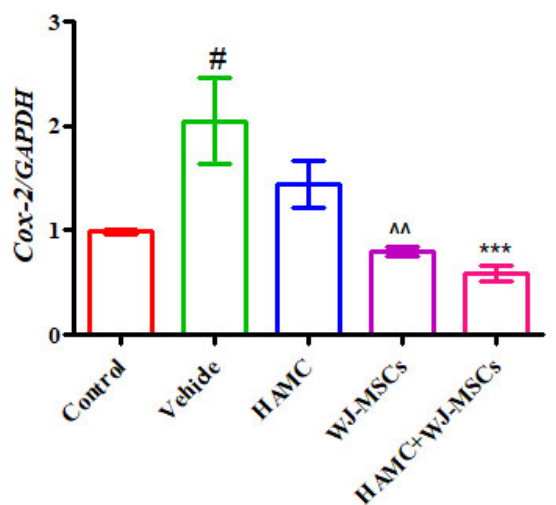

Figure 6. Combined injection of HAMC and WJ-MSC inhibits the mRNA expression of pro-inflammatory cytokine and matrix-degrading enzymes. (A) iNOS mRNA expression, (B) MMP-13 mRNA expression, (C) Adamts4 mRNA expression, and (D) Cox-2 mRNA expression. In the HAMC/WJ-MSCs-injected discs, mRNA expression of pro-inflammatory cytokine (inducible nitric oxide synthase; iNOS) and matrix-degrading enzymes, including $M M P-13$, A disintegrin and metalloproteinase with thrombospondin motifs 4 (Adamts4) and Cycloxygenase-2 (Cox-2) were found to be significantly downregulated compared to the vehicle-injected disc. Data are presented as mean $\pm \mathrm{SEM}$, one-way ANOVA followed by Tukey's post-hoc test. $(n=3)$. For time, glyceraldehyde 3-phosphate dehydrogenase (GAPDH) was used as internal control. ${ }^{\#} p<0.05,{ }^{\# \#} p<0.01,{ }^{\# \#} p<0.001$ (vehicle vs.control), $\$ p<0.05,{ }^{\$ \$} p<0.001$ (HAMC vs. vehicle), $p<0.05,{ }^{\wedge} p<0.001$ (WJ-MSCs vs. vehicle only), ${ }^{*} p<0.05,{ }^{* *} p<0.01,{ }^{* * *} p<0.001$ (HAMC + WJ-MSCs vs. vehicle only). 


\section{Discussion}

Cell therapy for IVD repair has gained considerable interest in the recent decade and direct implantation of MSCs has shown promising therapeutic potential [13]. There are, however, significant challenges, including the fact that the degenerated disc produces a harsh environment consisting of low glucose, low oxygen, low $\mathrm{pH}$ due to high lactic acid buildup, and low nutrients combined with an inflammatory milieu [3]. To overcome obstacles for successful disc repair, the selection of appropriate stem cells and the development of biomaterial-based cellular delivery systems that combine cells with cell-carrying matrix molecules such as fibrin and hyaluronan have become increasingly implemented [3]. Recently, various types of biomaterials have been reported to have the ability to prevent cell leakage and promote cell survival and stem cell differentiation toward the IVD cell phenotype [39]. Reports demonstrated that utilization of a carrier such as hyaluronan or annular sealant significantly prevented cell leakage, and the rate of osteophyte formation resulting from cell leakage has been reported to be less than $2.7 \%$ in rabbit IVD model [40]. Thus, the use of biomaterials has been strongly recommended to prevent leakage of implanted cells and to promote cell survival and prevent induction of unwanted differentiation of MSCs into osteoblasts [40]. However, the optimal biomaterial for IVD transplantation remains unknown. In our previous study, we demonstrated the efficacy of TissueFill (cross-linked 2\% (w/v) HA-based dermal filler) as a carrier of WJ-MSCs in a rabbit model of IVD degeneration [4]. TissueFill is commercialized dermal filler and a highly viscous hydrogel solution, but we chose TissueFill as a cell carrier due to the absence of clinically approved biomaterials for the treatment of IVD repair. When cultured in TissueFill, cell viability decreases and injection through a fine-gauge (26G) needle was difficult due to high viscosity and semi-diluted TissueFill was used because less viscous hydrogel was required to increase cell survival and injectability of the biomaterial.

In the recent decade, HAMC has been reported as a promising vehicle for delivery of various types of stem cells in many neurological disease models [24,25,36,41]. However, the HAMC hydrogel has not been tested in animal models of IVD degeneration. In the present study, we investigated the influence of HAMC on cell survival in vitro and thein vivo effects of HAMC loaded with WJ-MSCs in a rat model of IVD degeneration to test potential clinical usability of HAMC for cell delivery. Here, we used HAMC as a cell carrier because the HAMC gel could help prevent cell leakage, enhance cell attachment, exert an anti-inflammatory effect, and provide a favorable micro environment for IVD regeneration [40]. The primary findings of this study are as follows: (1) Our quality control assessment for WJ-MSCs demonstrated that WJ-MSCs showed a spindle fibroblast-like shape until fourteenth generations, which was consistent with our previous report [4]. Flow cytometric analysis of expressed surface antigens showed that these cells were uniformly positive for CD44, CD73, CD105, and CD90, and negative for the hematopoietic lineage markers CD45. Moreover, no structural or numerical chromosomal abnormalities were found in karyotype analyses of the cells until P14; (2) Cell viability in 3D HAMC was significantly greater than in TissueFill, indicating HAMC significantly promotes in-vitro cell survival; (3) Acute cell viability post-injection was significantly higher when cells were injected within an HAMC hydrogel compared to cells injected in TissueFill, suggesting that HAMC is more suitable for delivery of WJ-MSCs; (4) A single intradiscal injection of WJ-MSCs-loaded HAMC resulted in enhanced IVD regeneration compared with implantation of WJ-MSCs or HAMC alone, confirmed by radiological and histological findings-WJ-MSC-loaded HAMC showed the best regenerative effect by inhibiting pro-inflammatory cytokines and matrix degenerative enzymes.

In the in vitro cell viability assay, we observed a drop in cell viability following injection through a syringe with both HAMC and TissueFill; however, cell viability was improved compared to injection in PBS. This acute decrease was likely caused by the shear stress experienced by cells as they passed through the fine-gauge needle, as has been reported elsewhere [32-35], although the process of dissociating WJ-MSCs during the injection preparation may have also contributed. We also observed a decrease in WJ-MSC viability after 7 days of culture in HAMC or TissueFill, when compared with 2D standard culture, although the viability in HAMC was significantly greater than in TissueFill. The cells were cultured in the hydrogels in suspension with no additional media or supplements in 
order to challenge the cells with stress, which may have contributed to their decrease in viability over time. Furthermore, HAMC is reported to be approximately $90 \%$ degraded in vitro at 14 days [42], and therefore it is likely that by day 7 the mechanical strength of HAMC and TissueFill is reduced which may lead to reduced cell support.

In this study, we injected human WJ-MSCs into the rat xenograft model, but did not administer immunosuppressive agents based on the fact that WJ-MSCs has the weakest expression of immune related genes, including MHC II genes, Toll-like receptor 4 (TLR4), TLR3, Jagged1 (JAG1), neurogenic locus notch homolog protein 2 (NOTCH2), and NOTCH3 compared to MSCs isolated from other sources [40]. Our study showed that the HAMC/WJ-MSCs-implanted discs showed an overall decrease in inflammation of the disc. The HAMC hydrogel has been shown to reduce the inflammatory response in the CNS [26]. For example, intrathecal injection of the HAMC decreased the levels of IL-1 $\alpha$ in injured spinal cord due to anti-inflammatory properties of HA [26]. It has been also reported that the HA itself acts as an anti-inflammatory molecule by downregulating the expression of proinflammatory cytokines such as IFN- $\gamma$ and the apoptosis marker caspase-3 [43]. Our data demonstrate that the HAMC alone injected disc showed attenuated mRNA expression of $i N O S, M M P-13$, Adamts4, and Cox-2 and increased expression of aggrecan compared to vehicle alone injected disc (Figures 5 and 6), suggesting that combined injection of WJ-MSCs and HAMC promoted regenerative potential in degenerated disc.

Two key points to be addressed in future work include (1) by what mechanism implanted WJ-MSCs modulate the inflammatory microenvironment and vice versa, and (2) whether implanted WJ-MSCs would differentiate into NP cells. By incorporation of WJ-MSCs into a new biomaterial, HAMC could enhance cell survival by modulating inflammatory microenvironment and promote the ability to differentiate into the NP cell-like phenotype. In the present study, however, we did not track the implanted WJ-MSCS and could not confirm survival of implanted WJ-MSCS at 6 weeks post implantation. In terms of survival of implanted cells, there have been several reports on long-term survival (more than 3 months) of implanted MSCs loaded into biomaterials [44,45]. In our previous study, WJ-MSCs-loaded TissueFill (1\% cross-linked hyaluronic acid hydrogel) was intradiscally implanted into degenerated rabbit disc and no survived cells were found 12 weeks post implantation [4]. Here, we confirmed that a complex mixture of cytokines produced by WJ-MSCs, including TGF- $\beta$ ligands (TGF $\beta 1$, TGF $\beta 2$, and TGF $\beta 3$ ), growth differentiation factor-15 (GDF-15), chemokine (C-C motif) ligand 5 (CCL5), and MMP1, could trigger the multiple signaling system including TGF- $\beta$ signaling and stimulate IVD regeneration. WJ-MSCs are capable of immune modulation, immune suppression, and immune avoidance, making them optimal candidates for cellular therapies in allogenic transplantation [20]. WJ-MSCs have been extensively investigated as an anti-inflammatory agent, which is associated with their remarkable immunomodulatory effects $[46,47]$. Moreover, MSCs have been proven to be associated with suppression of lymphocyte activation. Similarly, MSCs express inducible nitric oxide synthase (iNOS) and indoleamine 2,3-dioxygenase (IDO) which leads to down-regulation of T cell proliferation in rodent and human MSCs, respectively [24,47,48]. To date, the anti-apoptotic property of stem cells seems to be the most widely established beneficial effect of MSCs $[47,49]$. In an earlier study, administration of MSCs displayed a renoprotective effect by preventing tissue apoptosis in an acute kidney injury (AKI) model. Briefly, MSC-treated AKI mice demonstrated the increased expression of the anti-apoptotic gene BCL2 and significant downregulation of the pro-apoptotic gene BAX [50].

Hence, the regenerative effects of HAMC/WJ-MSCs in our study might therefore be linked with immunomodulatory and anti-inflammatory effects via paracrine signaling, regardless of whether WJ-MSCs differentiated into NP-like cells. In addition, HAMC provided a superior carrier for WJ-MSCs and improved regeneration in a rat model of disc degeneration.

To the best of our knowledge, no other study has investigated the combined treatment of WJ-MSCs and HAMC hydrogel, to date. Previously, we investigated the efficacy of combined implantation of WJ-MSCs and TissueFill in a rabbit model of disc degeneration [4]. Similarly, we conducted a Phase 1 clinical trial by applying combined transplantation of autologous adipose-derived MSCs and TissueFill 
in chronic discogenic LBP patients: six patients among 10 participants showed improvement of pain and disability [1]. Herein, we have avoided the highly viscous nature of TissueFill by employing HAMC as an alternative, and based on our results, HAMC hydrogel was found to be superior to TissueFill, however, the explicit mechanism by which disc structure was restored remains to be investigated. It would be valuable to compare the in-vivo effects between TissueFill and HAMC hydrogel in future studies. Additionally, the regenerative effects of WJ-MSCs loaded HAMC must be tested using larger animal models to understand the feasibility of applying this approach to humans. Despite some limitations, this investigation demonstrates that regeneration following disc degeneration can be achieved by combined implantation of WJ-MSCs and HAMC.

\section{Materials and Methods}

\subsection{Preparation of Hydrogels}

Hyaluronan (HA, 1200-1900 kDa, Novamatrix, Drammen, Norway) and methylcellulose (MC, $300 \mathrm{kDa}$, Shin-Etsu, Tokyo, Japan) (Figure 1A) was used to prepare an injectable, biocompatible, and biodegradable hydrogel scaffold HAMC, as previously described [41]. Briefly, 24 hours before use, sterile-filtered HA and MC were dissolved into artificial cerebrospinal fluid (aCSF; R\&D Systems, Minneapolis, MN, USA), cell medium, or PBS at a concentration of $1 \% w / v$ and left at $4{ }^{\circ} \mathrm{C}$ overnight. Prior to use, HAMC was kept on ice for all experiments. TissueFill, a cross-linked HA commercially available hydrogel, was purchased and diluted to a $1 \%$ solution in aCSF or cell medium for use in in vitro experiments.

\subsection{Preparation and Quality Control of WJ-MSCs}

WJ-MSCs were purchased from the Good Manufacturing Practice (GMP) facility of CHA Biotech (Seongnamsi, Korea) and this study was conducted according to the World Medical Association Declaration of Helsinki. With the written informed consent and ethics committee approval (No. BD2014-055), umbilical cord was obtained immediately after birth.

WJ-MSCs were prepared as described elsewhere [4] and all culture conditions adhere to GMP standards. Briefly, the cord was washed with phosphate-buffered saline (PBS) and Wharton's jelly was then cut into pieces smaller than $5 \mathrm{~mm}^{3}$ after blood vessels were removed. The minced Wharton's jelly was digested for $6-10 \mathrm{~h}$ in a sterilized bottle with $15 \mathrm{~mL}$ culture medium containing collagenase of type I at $0.075 \%$ in $5 \%$ carbon dioxide, at $37{ }^{\circ} \mathrm{C}$ with agitation in an incubator. The cells were then washed three times with D-Hank's salt solution and centrifuged at $250 \times g$ for $10 \mathrm{~min}$ at room temperature, and the cells were resuspended in culture medium (Dulbecco's modified Eagle's medium; DMEM) with low glucose (DMEM-L; Gibco BRL) supplemented with 10\% (v/v) fetal bovine serum (FBS; Gibco BRL) and 1\% antibiotic-antimycotic solution (Gibco BRL) in humidified air with 5\% carbon dioxide at $37^{\circ} \mathrm{C}$. The cells were harvested after reaching $70-80 \%$ confluency and characterization tests were performed at passage 7. Quality control of these cells was performed according to the standards of the Korea's Ministry of Food and Drug Safety.

\subsection{In Vitro 3D Hydrogel Cell Survival}

WJ-MSCs were cultured in a serum-free Dulbecco's modified Eagle's medium (DMEM; Gibco BRL, Gaithersburg, MD, USA). On the day of the experiment, WJ-MSCs were dissociated using trypsin and $1 \times 10^{4}$ cells were suspended into either $150 \mathrm{uL}$ of $0.5 \%$ w/v HAMC in medium or $1 \%$ TissueFill or seeded in 2D with cell medium and pipetted into a 96-well plastic culture plate. Cells were cultured for 0, 1, 3, or 7 days and viability was assessed using calcein AM (for live cells) and ethidium homodimer (for dead cells) (ThermoFisher Waltham, MA, USA). Stained cells were imaged on a Zeiss 880 fluorescence microscope (Germany), and three fields of view per well were captured as a $\mathrm{z}$ stack approximately $1 \mathrm{~mm}$ in depth for the 3D hydrogel groups. The $\mathrm{z}$ stack was merged into a single image and the number of live cells per field of view was determined using ImageJ (La Jolla, CA, USA) and averaged across 
three wells within each biological replicate, with three replicates in total. Cell viability was expressed as the percentage of live cells at each time point normalized to the initial cell viability at time 0 .

\subsection{In Vitro Post-Injection Cell Viability}

Wharton's jelly-derived mesenchymal stem cells (WJ-MSCs) were cultured in a serum-free Dulbecco's modified Eagle's medium (DMEM; Gibco BRL, Gaithersburg, MD, USA). On the day of the experiment, WJ-MSCs were trypsinized and $2 \times 10^{4}$ cells/uL were suspended into equal volume of $1 \%$ HAMC, final concentration $0.5 \% \mathrm{HA} / 0.5 \% \mathrm{MC}, w / v, 1 \%$ TissueFill, or cell medium and loaded into a Hamilton $10 \mu \mathrm{L}$ Gastight syringe \#1701 fitted with a $26 \mathrm{G} 45^{\circ}$ beveled needle. Injections were performed to mimic the in vivo cell delivery protocol by injecting $50 \mathrm{uL}$ into a 96-well plate in triplicate. Immediately following injection, $200 \mathrm{uL}$ of cell medium was pipetted on top of the cells. To assess post-injection cell viability, $20 \mathrm{uL}$ of CCK-8 (Sigma-Aldrich, St Louis, MO, USA) was added to each well and incubated for 90 minutes. A spectrophotometer was used to measure optical density at $450 \mathrm{~nm}$ of each well to determine relative cell viability. Viability was expressed as optical density normalized to viability at time 0 .

\subsection{Injury-Induced Disc Degeneration Rat Model}

The animal experiments were performed as directed and approved by the Institutional Animal Care and Use Committee (IACUC) of CHA Bundang Medical Center (IACUC180189). Six-week-old female Sprague-Dawley rats (220-240 g) were purchased from Orient Bio Inc., Korea, and were acclimatized for a week at a life/dark cycle of $12 / 12 \mathrm{~h}$ (temperature; $22 \pm 1{ }^{\circ} \mathrm{C}$ and relative humidity; $50 \% \pm 1 \%$ ) and free access to food and water ad libitum.

Prior surgery, rats were deeply anesthetized with general anesthesia mixture of Zoletil ${ }^{\circledR}(50 \mathrm{mg} / \mathrm{kg}$ Virbac Laboratories, France) and Rompun ${ }^{\circledR}(10 \mathrm{mg} / \mathrm{kg}$, Bayer, Korea) injected intraperitoneally. Thereafter, the proximal most part of the tail along with the pelvic area was sterilized with $70 \%$ alcohol followed by povidone iodine. A 1-cm longitudinal incision was made along the tail exposing the lateral portion of the coccygeal disc. Following that, a 21G sterile needle was inserted into the center of the coccygeal discs (Co6-7, Co7-8). To prevent further damage, needle insertion was limited to $5 \mathrm{~mm}$ in depth. Following which, spinal needle was rotated at complete 360 degree and kept in place for $30 \mathrm{~s}$. Finally, the skin was sutured, disinfected and as a prophylactic intervention, $0.9 \%$ sterile normal saline $(5 \mathrm{~mL})$ was injected subcutaneously, and appropriate dose of analgesic (Ketoprofen, SCD Pharm. Co. Ltd., Korea) and antibiotic (Cefazolin, CKD Pharmaceuticals, Korea) for 3 days after surgery. During procedure, body temperature of rats was maintained at $37^{\circ} \mathrm{C}$ with the help of thermostatically regulated heating pad.

\subsection{Experimental Design in a Rat Disc Degeneration Model}

Our data also showed that WJ-MSCs maintained high viability and pluripotency without any karyotype abnormalities until the nineteenth passage (Figure S1). The seventh passage of WJ MSCs was therefore used for our study.

Forty rats were randomly divided into four groups: (1) PBS vehicle, (2) HAMC, (3) WJ-MSCs $\left(2 \times 10^{4}\right.$ cells), and (4) WJ-MSCs/ HAMC ( $n=10$ /each group) treated groups. Thenormal discs (Co5-6) proximal to the treated discs in each tail were used as a control. Two weeks following disc injury, ten animals per group received either PBS only (vehicle group), WJ-MSCs (seventh passages, $2 \times 10^{4}$ cells $/ 2 \mu \mathrm{L} /$ disc), referred to as WJ-MSC group, HAMC $(2 \mu \mathrm{L} 1 \%$ HAMC with PBS $=0.5 \%$ HAMC $)$ and WJ-MSCs-loaded HAMC $\left(1 \mu \mathrm{L}\right.$ of $2 \times 10^{4}$ cells $+1 \mu \mathrm{L} 1 \%$ HAMC $=0.5 \%$ HAMC (HAMC/WJ-MSCs group). Coccygeal discs were removed following sacrifice 6 weeks after implantation for radiologic and histologic analysis. 


\subsection{Magnetic Resonance Imaging (MRI)}

Six weeks after implantation, we performed 9.4 T MRI (Bruker BioSpec, USA) to compare degree of coccygeal disc degeneration and water content of the disc. T2-weighted imaging protocol was set as: (1) coronal plane; time to repetition (TR) of $5000 \mathrm{~ms}$, time to echo (TE) of $30 \mathrm{~ms}$, 150 horizontal $\times 150$ vertical matrix; field of view of 15 horizontal $\times 15$ vertical, and $0.5 \mathrm{~mm}$ slices with $0 \mathrm{~mm}$ spacing between each slice. (2) Sagittal plane; time to repetition (TR) of 5000 milliseconds (ms), time to echo (TE) of $50 \mathrm{~ms}, 200$ horizontal $\times 600$ vertical matrix; field of view of 20 horizontal $\times 60$ vertical, and $0.8 \mathrm{~mm}$ slices with $0 \mathrm{~mm}$ spacing between each slice. The signal intensity and MRI index (the area of NP multiplied by average signal intensity) were evaluated [51]. The region of interest (ROI) was defined as high signal intensity area in the mid coronal plane of the T2-weighted images; as the outline of the NP, measured the ROI using Image J software (the National Institutes of Health, Bethesda, MD, USA) [51]. The measurement of MRI index was performed by two independent observers who were blinded to the specimen's treatment.

\subsection{RNA Isolation and Real Time RT-PCR}

Six weeks after implantation, the coccygeal discs were harvested and approximately $100 \mathrm{mg} \mathrm{NP}$ was isolated from the disc and triturated under liquid nitrogen in a pre-cooled mortar. Liquid nitrogen was allowed to volatilize and with the help of pestle, hardened nucleus pulposus was ground into a powder. Viscous NP was then incubated in $1 \mathrm{~mL}$ Trizol (Invitrogen, Carlsbad, CA, USA) at $37^{\circ} \mathrm{C}$ for $10 \mathrm{~min}$. Chloroform $(0.2 \mathrm{~mL})$ was then added, and centrifuged at $12,000 \mathrm{~g}$ and $4{ }^{\circ} \mathrm{C}$ for $15 \mathrm{~min}$. Following centrifugation, the top layer was transferred to $0.5 \mathrm{~mL}$ isopropanol for precipitation and centrifuged again. Next the supernatant was removed, and RNA precipitate was washed once with $75 \%$ alcohol and dried at $37^{\circ} \mathrm{C}$ for $10 \mathrm{~min}$. Then the RNA was dissolved with $20 \mu \mathrm{L}$ RNase-free water. For PCR analysis, $1 \mu \mathrm{g}$ of RNA was reverse transcribed to complementary DNA (cDNA) using an RT PreMix Kit (Seoul, Korea). Real time PCR was performed by using SYBR green master mix and mRNA expression was analyzed by ABI Step one Real-time PCR system (Applied Biosystems, UK). Primer sequences for the gene of interest used in this study are given in Table 1 . The typical PCR amplification profile used was denaturation at $95^{\circ} \mathrm{C}$ for $10 \mathrm{~min}$ followed by a second step at $95^{\circ} \mathrm{C}$ for $15 \mathrm{~s}$, followed by annealing and extension at $60^{\circ} \mathrm{C}$ for $30 \mathrm{~s}$ and melting curve analysis at 40 cycles, in which dissociation curve software was used to ensure that only a single product was amplified. Target genes were normalized with glyceraldehyde 3-phosphate dehydrogenase (GAPDH) and data were analyzed by $2^{-\Delta \Delta c t}$ method.

Table 1. Primer sequences for references and target genes.

\begin{tabular}{|c|c|c|}
\hline Primers & Direction & Sequences \\
\hline \multirow{2}{*}{ Adamts4 } & Forward & 5'-CGTGGTGTGTGTGTGTGT-3' \\
\hline & Reverse & 5'- AGAGGAAAGTAGGGCAGGT-3' \\
\hline \multirow{2}{*}{$\operatorname{Cox}-2$} & Forward & 5'-TGTATGCTACCATCTGGCTTCGG-3' \\
\hline & Reverse & 5'-GTTTGGAACAFTCGCTCGTCATC-3' \\
\hline \multirow{2}{*}{$M M P-13$} & Forward & 5'-TGGTCCCTGCCCCTTCCCTA-3' \\
\hline & Reverse & 5'-CCGCAAGAGTCACAGGATGGTAGTA-3' \\
\hline \multirow{2}{*}{$i N O S$} & Forward & 5'-CTGCAGGTCTTTGACGCTCGGAG -3' \\
\hline & Reverse & 5'-GTGGAACACAGGGGTGATGCTCC-3' \\
\hline \multirow{2}{*}{ GAPDH } & Forward & 5'-CAАСТСССТСAAGATTGTCAGCAA-3' \\
\hline & Reverse & 5'-GGCATGGACTGTGGTCATGA-3' \\
\hline
\end{tabular}

Inducible nitric oxide synthase, $i$ NOS; matrix metalloproteinase-13, MMP-13; A disintegrin and metalloproteinase with thrombospondin motifs 4, Adamts4; Cycloxygenase-2, Cox-2; glyceraldehyde 3-phosphate dehydrogenase, GAPDH. 


\subsection{Safranin-O Staining}

Six weeks after implantation, rats were euthanized and discs from each rat were harvested for histological analysis. Following which, discs with adjacent vertebral body were fixed in $10 \%$ neutral buffered formalin for one week, decalcified in RapidCal Immuno (BBC Biochemical, Mount Vernon, WA, USA) for 2 weeks. Tissues were then processed for paraffin embedding and sectioning into coronal sections $(10 \mu \mathrm{m})$ using microtome (Leica). The sections were dewaxed, rehydrated, and stained with Safranin-O (Sigma, USA) to evaluate the quantity and distribution range of proteoglycans. Then, sections were mounted using mounting media and scanned with an OLYMPUS C-mount camera adapter (U-TVO.63XC, Tokyo, Japan). All samples were subjectively assessed for tissue morphology and architecture by pathologists who were blinded to the sample information. For the assessment of histologic structure, a 14-point score was used based on safranin-O staining [52]. At 6 weeks post-implantation, the scoring consists of five parameters: NP structure, NP clefts/fissures, annulus fibrosus (AF)/NP boundary, AF structure, and AF/clefts/fissures. The sum of the separate scores ranges from 0 (normal) to 14 (most severe) [52].

\subsection{Immunohistochemistry}

Six weeks following implantation, rats were euthanized via carbon dioxide inhalation and coccygeal discs were collected, and immunohistochemical analysis was performed for aggrecan, collagen type II, and MMP-13. Briefly, explants were fixed overnight in a $4 \%$ paraformaldehyde (PFA) solution and decalcified in a decalcification solution; RapidCal Immuno (BBC Biochemical, Mount Vernon, WA, USA) for 2 weeks. Thereafter, discs were embedded within paraffin wax and sectioned longitudinally using a microtome (Leica) into 5- to 10- $\mu \mathrm{m}$ thickness sections. Prior to immunohistochemical staining, sections were dewaxed, rehydrated, and stained with antibodies against aggrecan (1:1000, Abcam, UK), collagen type II (1:100, Abcam, UK) and MMP-13 (1:200, Abcam, UK). After 24 hours, sections were washed with phosphate buffered saline with Tween 20 and incubated with the secondary antibody anti-Rb horseradish peorixdase (Roche Diagnostics Ltd., Switzerland), and Alexa 488-conjugated secondary antibodies (Invitrogen, USA). After a washing step, the specimens were counterstained with DAPI (1:500, Abcam, UK) for $10 \mathrm{~min}$. Finally, the sections were mounted and examined using a fluorescence microscope (Zeiss 880, Germany and Leica SP5, Germany). The number of positive cells was counted in three random fields $(n=4$ per group) $(\times 40)$ using Image J software (https://imagej.nih.gov/ij/).

\subsection{Statistical Analysis}

Data were analyzed by using GraphPad Prism (version 5.01, GraphPad Software). Data are presented as mean \pm standard error of the mean (SEM) unless otherwise stated. In vitro cell viability data were analyzed by two-way ANOVA, followed by Tukey's post-hoc test $p<0.05$. PCR and immunofluorescence data were analyzed using one-way ANOVA, followed by Tukey's post-hoc test. $p$-values $<0.05$ were considered statistically significant.

\section{Conclusions}

In conclusion, this study addresses the efficacy of combined implantation of WJ-MSCs and HAMC for IVD regeneration. Combined injection of WJ-MSCs and HAMC enhanced IVD regeneration through increase in cell survival, attenuation of the activation of iNOS, MMP-13, ADAMTS4 and COX-2, and significant up-regulation of ECM, such as aggrecan and collagen type II. This strategy offers an advantage for clinical application because WJ-MSCs-loaded HAMC induces IVD repair more effectively than cell injection alone and supports the potential clinical use of HAMC for cell delivery to attenuate IVD degeneration and promote regeneration. 
Supplementary Materials: Supplementary materials can be found at http://www.mdpi.com/1422-0067/21/19/ 7391/s1. (A) Morphology of WJ-MSCs in the second, fourth, and seventh generation. P2, second generation; P4; fourth generation; P7; seventh generation (lens: $\times 40, \times 100$ and $\times 200$ respectively). (B) Cellular multipotent differentiation of WJ-MSCs. Following adipogenic induction for 14 days, WJ-MSCs were positive for Oil red-O staining. Following osteogenic and chondrogenic induction for 14 days, WJ-MSCs were positive for alizarin red staining and alcian blue staining, respectively. Scale bars, $10 \mu \mathrm{m}$. (C) cell population doubling time. (D) Flow cytometric analysis of cell surface markers of WJ-MSCs at P7. (E) Chromosome karyotype analysis of the cultured WJ-MSCs.

Author Contributions: Conceptualization, U.Y.C., H.P.J., S.P., M.J.C., M.S.S. and I.H.; data curation, U.Y.C., H.P.J., S.P. and E.J.R.; formal analysis, U.Y.C., H.P.J., K.T.K., J.W.K., H.C., M.J.C., S.Y.K., S.S. and I.H.; funding acquisition, M.S.S. and I.H.; investigation, U.Y.C., H.P.J., S.P., K.T.K., J.W.K., H.C., S.Y.K., E.J.R. and S.S.; methodology, J.W.K.; supervision, M.S.S. and I.H.; validation, K.T.K. and M.S.S.; writing-original draft, H.P.J., S.P. and I.H.; writing-review and editing, I.H. All authors have read and agreed to the published version of the manuscript.

Funding: This research was supported by the Korea Health Technology Research and Development Project, Ministry for Health and Welfare Affairs (HR16C0002, HI16C0106, and HI20C0579).

Acknowledgments: The authors gratefully acknowledge the support of the Shoichet laboratory, University of Toronto, Ontario, Canada.

Conflicts of Interest: The authors declare no conflict of interest.

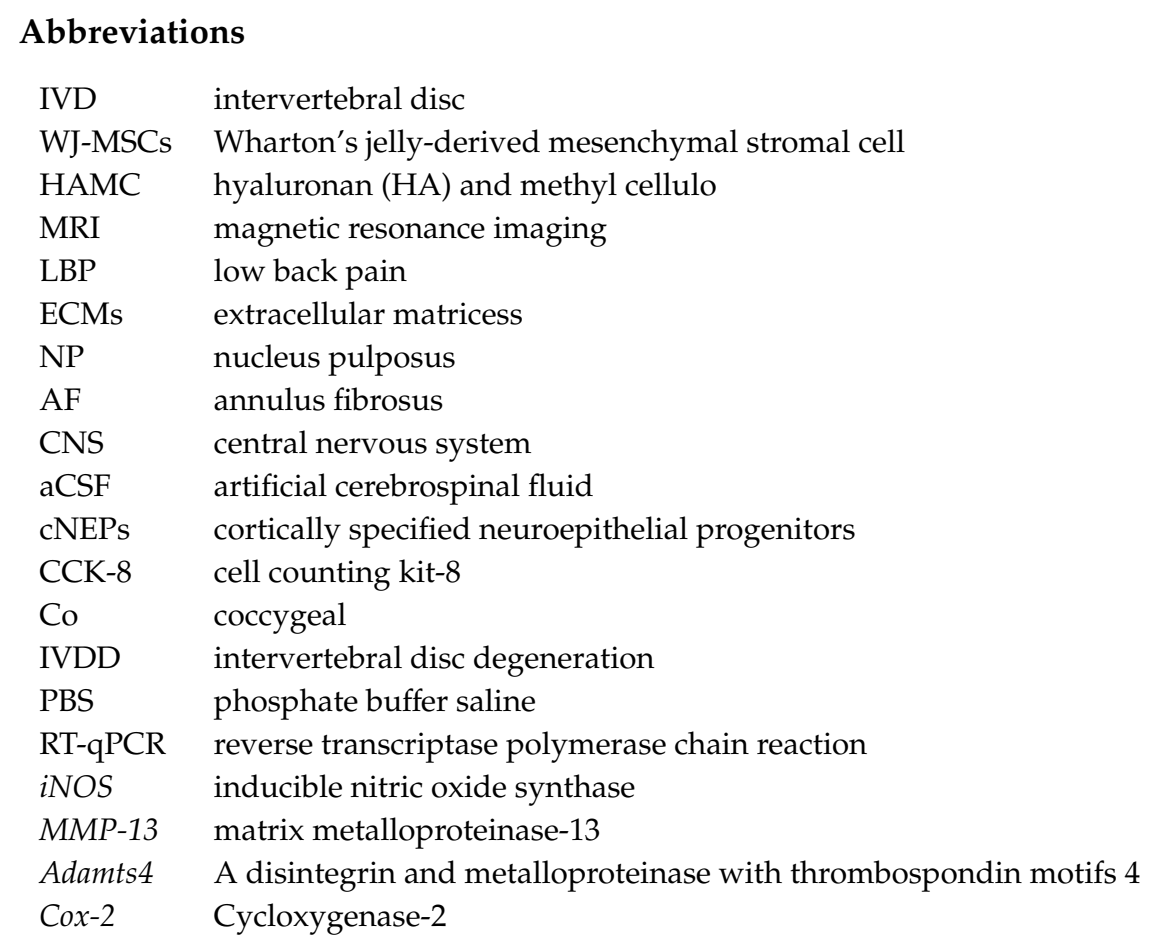

\section{References}

1. Kumar, H.; Ha, D.-H.; Lee, E.-J.; Park, J.H.; Shim, J.H.; Ahn, T.-K.; Kim, K.-T.; Ropper, A.E.; Sohn, S.; Kim, C.-H. Safety and tolerability of intradiscal implantation of combined autologous adipose-derived mesenchymal stem cells and hyaluronic acid in patients with chronic discogenic low back pain: 1-year follow-up of a phase I study. Stem Cell Res. Ther. 2017, 8, 1-14. [CrossRef] [PubMed]

2. Takeoka, Y.; Yurube, T.; Nishida, K. Gene therapy approach for intervertebral disc degeneration: An update. Neurospine 2020, 17, 3. [CrossRef] [PubMed]

3. Han, I.; Ropper, A.E.; Konya, D.; Kabatas, S.; Toktas, Z.; Aljuboori, Z.; Zeng, X.; Chi, J.H.; Zafonte, R.; Teng, Y.D. Biological approaches to treating intervertebral disk degeneration: Devising stem cell therapies. Cell Transplant. 2015, 24, 2197-2208. [CrossRef] [PubMed]

4. Ahn, J.; Park, E.-M.; Kim, B.J.; Kim, J.-S.; Choi, B.; Lee, S.-H.; Han, I. Transplantation of human Wharton's jelly-derived mesenchymal stem cells highly expressing TGF $\beta$ receptors in a rabbit model of disc degeneration. Stem Cell Res. Ther. 2015, 6, 190. [CrossRef] [PubMed] 
5. Meisel, H.-J.; Agarwal, N.; Hsieh, P.C.; Skelly, A.; Park, J.-B.; Brodke, D.; Wang, J.C.; Yoon, S.T.; Buser, Z. Cell therapy for treatment of intervertebral disc degeneration: A systematic review. Glob. Spine J. 2019, 9, 39S-52S. [CrossRef] [PubMed]

6. Silva, A.J.; Corte-Real, J.V.; Ferreira, J.R.; Cunha, C.; Gonçalves, M.; Barbosa, M.A.; Santos, S.G.; Gonçalves, R.M. Macrophages down-regulate gene expression of intervertebral disc degenerative markers under a pro-inflammatory microenvironment. Front. Immunol. 2019, 10, 1508. [CrossRef]

7. Kraemer, J. Natural course and prognosis of intervertebral disc diseases. International Society for the Study of the Lumbar Spine Seattle, Washington, June 1994. Spine 1995, 20, 635-639.

8. Antoniou, J.; Steffen, T.; Nelson, F.; Winterbottom, N.; Hollander, A.P.; Poole, R.A.; Aebi, M.; Alini, M. The human lumbar intervertebral disc: Evidence for changes in the biosynthesis and denaturation of the extracellular matrix with growth, maturation, ageing, and degeneration. J. Clin. Investig. 1996, 98, 996-1003. [CrossRef] [PubMed]

9. Larson, J.W., III; Levicoff, E.A.; Gilbertson, L.G.; Kang, J.D. Biologic modification of animal models of intervertebral disc degeneration. JBJS 2006, 88 Suppl. 2, 83-87. [CrossRef]

10. Peng, B.G. Pathophysiology, diagnosis, and treatment of discogenic low back pain. World J. Orthop. 2013, 4, 42-52. [CrossRef]

11. Wang, F.; Cai, F.; Shi, R.; Wang, X.H.; Wu, X.T. Aging and age related stresses: A senescence mechanism of intervertebral disc degeneration. Osteoarthr. Cartil. 2016, 24, 398-408. [CrossRef]

12. Isa, I.L.M.; Abbah, S.A.; Kilcoyne, M.; Sakai, D.; Dockery, P.; Finn, D.P.; Pandit, A. Implantation of hyaluronic acid hydrogel prevents the pain phenotype in a rat model of intervertebral disc injury. Sci. Adv. 2018, 4, eaaq0597.

13. Sakai, D.; Andersson, G.B. Stem cell therapy for intervertebral disc regeneration: Obstacles and solutions. Nat. Rev. Rheumatol. 2015, 11, 243-256. [CrossRef] [PubMed]

14. Nesti, L.J.; Li, W.-J.; Shanti, R.M.; Jiang, Y.J.; Jackson, W.; Freedman, B.A.; Kuklo, T.R.; Giuliani, J.R.; Tuan, R.S. Intervertebral disc tissue engineering using a novel hyaluronic acid-nanofibrous scaffold (HANFS) amalgam. Tissue Eng. Part A 2008, 14, 1527-1537. [CrossRef] [PubMed]

15. Nekanti, U.; Rao, V.B.; Bahirvani, A.G.; Jan, M.; Totey, S.; Ta, M. Long-term expansion and pluripotent marker array analysis of Wharton's jelly-derived mesenchymal stem cells. Stem Cells Dev. 2010, 19, 117-130. [CrossRef] [PubMed]

16. Liu, S.; Hou, K.D.; Yuan, M.; Peng, J.; Zhang, L.; Sui, X.; Zhao, B.; Xu, W.; Wang, A.; Lu, S. Characteristics of mesenchymal stem cells derived from Wharton's jelly of human umbilical cord and for fabrication of non-scaffold tissue-engineered cartilage. J. Biosci. Bioeng. 2014, 117, 229-235. [CrossRef]

17. Mallis, P.; Michalopoulos, E.; Chatzistamatiou, T.; Stavropoulos-Giokas, C. Mesenchymal stromal cells as potential immunomodulatory players in severe acute respiratory distress syndrome induced by SARS-CoV-2 infection. World J. Stem Cells 2020, 12, 731-751. [CrossRef]

18. K Batsali, A.; Kastrinaki, M.-C.; A Papadaki, H.; Pontikoglou, C. Mesenchymal stem cells derived from Wharton's Jelly of the umbilical cord: Biological properties and emerging clinical applications. Curr. Stem Cell Res. Ther. 2013, 8, 144-155. [CrossRef]

19. Jyothi Prasanna, S.; Sowmya Jahnavi, V. Wharton's jelly mesenchymal stem cells as off-the-shelf cellular therapeutics: A closer look into their regenerative and immunomodulatory properties. Open Tissue Eng. Regen. Med. J. 2011, 4, 28-38. [CrossRef]

20. Marino, L.; Castaldi, M.A.; Rosamilio, R.; Ragni, E.; Vitolo, R.; Fulgione, C.; Castaldi, S.G.; Serio, B.; Bianco, R.; Guida, M. Mesenchymal Stem Cells from the Wharton's Jelly of the Human Umbilical Cord: Biological Properties and Therapeutic Potential. Int. J. Stem Cells 2019, 12, 218. [CrossRef]

21. Prasanna, S.J.; Gopalakrishnan, D.; Shankar, S.R.; Vasandan, A.B. Pro-inflammatory cytokines, IFN $\gamma$ and $\mathrm{TNF} \alpha$, influence immune properties of human bone marrow and Wharton jelly mesenchymal stem cells differentially. PLoS ONE 2010, 5, e9016. [CrossRef]

22. Kim, D.-W.; Staples, M.; Shinozuka, K.; Pantcheva, P.; Kang, S.-D.; Borlongan, C.V. Wharton's jelly-derived mesenchymal stem cells: Phenotypic characterization and optimizing their therapeutic potential for clinical applications. Int. J. Mol. Sci. 2013, 14, 11692-11712. [CrossRef] [PubMed]

23. Caicco, M.J.; Zahir, T.; Mothe, A.J.; Ballios, B.G.; Kihm, A.J.; Tator, C.H.; Shoichet, M.S. Characterization of hyaluronan-methylcellulose hydrogels for cell delivery to the injured spinal cord. J. Biomed. Mater. Res. Part A 2013, 101, 1472-1477. [CrossRef] [PubMed] 
24. Payne, S.L.; Tuladhar, A.; Obermeyer, J.M.; Varga, B.V.; Teal, C.J.; Morshead, C.M.; Nagy, A.; Shoichet, M.S. Initial cell maturity changes following transplantation in a hyaluronan-based hydrogel and impacts therapeutic success in the stroke-injured rodent brain. Biomaterials 2019, 192, 309-322. [CrossRef] [PubMed]

25. Mitrousis, N.; Hacibekiroglu, S.; Ho, M.T.; Sauvé, Y.; Nagy, A.; van der Kooy, D.; Shoichet, M.S. Hydrogel-mediated co-transplantation of retinal pigmented epithelium and photoreceptors restores vision in an animal model of advanced retinal degeneration. Biomaterials 2020, 257, 120233. [CrossRef]

26. Ho, M.T.; Teal, C.J.; Shoichet, M.S. A hyaluronan/methylcellulose-based hydrogel for local cell and biomolecule delivery to the central nervous system. Brain Res. Bull. 2019, 148, 46-54. [CrossRef]

27. Cooke, M.J.; Wang, Y.; Morshead, C.M.; Shoichet, M.S. Controlled epi-cortical delivery of epidermal growth factor for the stimulation of endogenous neural stem cell proliferation in stroke-injured brain. Biomaterials 2011, 32, 5688-5697. [CrossRef]

28. Kang, C.E.; Poon, P.C.; Tator, C.H.; Shoichet, M.S. A new paradigm for local and sustained release of therapeutic molecules to the injured spinal cord for neuroprotection and tissue repair. Tissue Eng. Part A 2009, 15, 595-604. [CrossRef]

29. Ballios, B.G.; Cooke, M.J.; van der Kooy, D.; Shoichet, M.S. A hydrogel-based stem cell delivery system to treat retinal degenerative diseases. Biomaterials 2010, 31, 2555-2564. [CrossRef]

30. Austin, J.W.; Kang, C.E.; Baumann, M.D.; DiDiodato, L.; Satkunendrarajah, K.; Wilson, J.R.; Stanisz, G.J.; Shoichet, M.S.; Fehlings, M.G. The effects of intrathecal injection of a hyaluronan-based hydrogel on inflammation, scarring and neurobehavioural outcomes in a rat model of severe spinal cord injury associated with arachnoiditis. Biomaterials 2012, 33, 4555-4564. [CrossRef]

31. Wang, Y.; Lapitsky, Y.; Kang, C.E.; Shoichet, M.S. Accelerated release of a sparingly soluble drug from an injectable hyaluronan-methylcellulose hydrogel. J. Control. Release 2009, 140, 218-223. [CrossRef]

32. Aguado, B.A.; Mulyasasmita, W.; Su, J.; Lampe, K.J.; Heilshorn, S.C. Improving viability of stem cells during syringe needle flow through the design of hydrogel cell carriers. Tissue Eng. Part A 2012, 18, 806-815. [CrossRef] [PubMed]

33. Connolly, S.; McGourty, K.; Newport, D. The in vitro inertial positions and viability of cells in suspension under different in vivo flow conditions. Sci. Rep. 2020, 10, 1-13. [CrossRef] [PubMed]

34. Rossetti, T.; Nicholls, F.; Modo, M. Intracerebral cell implantation: Preparation and characterization of cell suspensions. Cell Transplant. 2016, 25, 645-664. [CrossRef] [PubMed]

35. Wahlberg, B.; Ghuman, H.; Liu, J.R.; Modo, M. Ex vivo biomechanical characterization of syringe-needle ejections for intracerebral cell delivery. Sci. Rep. 2018, 8, 1-17. [CrossRef] [PubMed]

36. Payne, S.L.; Anandakumaran, P.N.; Varga, B.V.; Morshead, C.M.; Nagy, A.; Shoichet, M.S. In vitro maturation of human iPSC-derived neuroepithelial cells influences transplant survival in the stroke-injured rat brain. Tissue Eng. Part A 2018, 24, 351-360. [CrossRef] [PubMed]

37. Takeoka, Y.; Yurube, T.; Morimoto, K.; Kunii, S.; Kanda, Y.; Tsujimoto, R.; Kawakami, Y.; Fukase, N.; Takemori, T.; Omae, K. Reduced nucleotomy-induced intervertebral disc disruption through spontaneous spheroid formation by the Low Adhesive Scaffold Collagen (LASCol). Biomaterials 2020, 235, 119781. [CrossRef] [PubMed]

38. Le Maitre, C.L.; Freemont, A.J.; Hoyland, J.A. Accelerated cellular senescence in degenerate intervertebral discs: A possible role in the pathogenesis of intervertebral disc degeneration. Arthritis Res. Ther. 2007, 9, R45. [CrossRef] [PubMed]

39. Peng, Y.; Huang, D.; Liu, S.; Li, J.; Qing, X.; Shao, Z. Biomaterials-Induced Stem Cells Specific Differentiation Into Intervertebral Disc Lineage Cells. Front. Bioeng. Biotechnol. 2020, 8, 56. [CrossRef]

40. Yim, R.L.-H.; Lee, J.T.-Y.; Bow, C.H.; Meij, B.; Leung, V.; Cheung, K.M.; Vavken, P.; Samartzis, D. A systematic review of the safety and efficacy of mesenchymal stem cells for disc degeneration: Insights and future directions for regenerative therapeutics. Stem Cells Dev. 2014, 23, 2553-2567. [CrossRef]

41. Ballios, B.G.; Cooke, M.J.; Donaldson, L.; Coles, B.L.; Morshead, C.M.; van der Kooy, D.; Shoichet, M.S. A hyaluronan-based injectable hydrogel improves the survival and integration of stem cell progeny following transplantation. Stem Cell Rep. 2015, 4, 1031-1045. [CrossRef]

42. Gupta, D.; Tator, C.H.; Shoichet, M.S. Fast-gelling injectable blend of hyaluronan and methylcellulose for intrathecal, localized delivery to the injured spinal cord. Biomaterials 2006, 27, 2370-2379. [CrossRef] [PubMed] 
43. Kazezian, Z.; Sakai, D.; Pandit, A. Hyaluronic acid microgels modulate inflammation and key matrix molecules toward a regenerative signature in the injured annulus fibrosus. Adv. Biosyst. 2017, 1, 1700077. [CrossRef] [PubMed]

44. Sobajima, S.; Vadala, G.; Shimer, A.; Kim, J.S.; Gilbertson, L.G.; Kang, J.D. Feasibility of a stem cell therapy for intervertebral disc degeneration. Spine J. 2008, 8, 888-896. [CrossRef] [PubMed]

45. Sakai, D.; Mochida, J.; Yamamoto, Y.; Nomura, T.; Okuma, M.; Nishimura, K.; Nakai, T.; Ando, K.; Hotta, T. Transplantation of mesenchymal stem cells embedded in Atelocollagen ${ }^{\circledR}$ gel to the intervertebral disc: A potential therapeutic model for disc degeneration. Biomaterials 2003, 24, 3531-3541. [CrossRef]

46. Gao, F.; Chiu, S.; Motan, D.; Zhang, Z.; Chen, L.; Ji, H.; Tse, H.; Fu, Q.-L.; Lian, Q. Mesenchymal stem cells and immunomodulation: Current status and future prospects. Cell Death Dis. 2016, 7, e2062. [CrossRef] [PubMed]

47. Lee, K.-H.; Tseng, W.-C.; Yang, C.-Y.; Tarng, D.-C. The anti-inflammatory, anti-oxidative, and anti-apoptotic benefits of stem cells in acute ischemic kidney injury. Int. J. Mol. Sci. 2019, 20, 3529. [CrossRef]

48. Su, J.; Chen, X.; Huang, Y.; Li, W.; Li, J.; Cao, K.; Cao, G.; Zhang, L.; Li, F.; Roberts, A. Phylogenetic distinction of iNOS and IDO function in mesenchymal stem cell-mediated immunosuppression in mammalian species. Cell Death Differ. 2014, 21, 388-396. [CrossRef]

49. Rowart, P.; Erpicum, P.; Detry, O.; Weekers, L.; Grégoire, C.; Lechanteur, C.; Briquet, A.; Beguin, Y.; Krzesinski, J.-M.; Jouret, F. Mesenchymal stromal cell therapy in ischemia/reperfusion injury. J. Immunol. Res. 2015, 2015, 602597. [CrossRef]

50. Bianchi, F.; Sala, E.; Donadei, C.; Capelli, I.; La Manna, G. Potential advantages of acute kidney injury management by mesenchymal stem cells. World J. Stem Cells 2014, 6, 644. [CrossRef]

51. Hua, J.; Liang, C.; Yu, R.; Zhang, M. In The relationship between MRI and histology in a rat model of intervertebral disc degeneration. Proc. Intl. Soc. Mag. Reson. Med 2014, 22, 1253.

52. Tam, V.; Chan, W.C.W.; Leung, V.Y.L.; Cheah, K.S.E.; Cheung, K.M.C.; Sakai, D.; McCann, M.R.; Bedore, J.; Séguin, C.A.; Chan, D. Histological and reference system for the analysis of mouse intervertebral disc. J. Orthop. Res. 2018, 36, 233-243. [CrossRef] [PubMed]

(C) 2020 by the authors. Licensee MDPI, Basel, Switzerland. This article is an open access article distributed under the terms and conditions of the Creative Commons Attribution (CC BY) license (http://creativecommons.org/licenses/by/4.0/). 